\title{
Smith Normal Form and acyclic matrices
}

\author{
In-Jae Kim • Bryan L. Shader
}

Received: 19 August 2005 / Accepted: 14 January 2008 / Published online: 31 January 2008

(C) Springer Science+Business Media, LLC 2008

\begin{abstract}
An approach, based on the Smith Normal Form, is introduced to study the spectra of symmetric matrices with a given graph. The approach serves well to explain how the path cover number (resp. diameter of a tree $T$ ) is related to the maximal multiplicity MaxMult $(T)$ occurring for an eigenvalue of a symmetric matrix whose graph is $T$ (resp. the minimal number $q(T)$ of distinct eigenvalues over the symmetric matrices whose graphs are $T$ ). The approach is also applied to a more general class of connected graphs $G$, not necessarily trees, in order to establish a lower bound on $q(G)$.
\end{abstract}

Keywords Smith Normal Form · Acyclic matrix · Graph spectra

\section{Introduction}

This paper concerns the relationship between the algebraic and geometric properties of a symmetric matrix and the combinatorial arrangement of its nonzero entries (i.e., its graph). We begin by establishing some basic graph theoretic notation and terminology that follows that in [4].

A graph $G$ consists of a vertex set $V(G)$ and an edge set $E(G)$, where an edge is an unordered pair of distinct vertices of $G$. We use $u v$ to denote the edge joining vertices $u$ and $v$. If $u v$ is an edge, then we say that $u$ and $v$ are adjacent, and that $v$ is a neighbor of $u$. A vertex is incident with an edge if it is one of the two vertices

I.-J. Kim

Department of Mathematics and Statistics, Minnesota State University, Mankato,

MN 56001, USA

B.L. Shader (凶)

Department of Mathematics, University of Wyoming, Laramie, WY 82071, USA

e-mail: bshader@uwyo.edu 
of the edge. The degree of a vertex is the number of edges incident to the vertex. A subgraph of a graph $G$ is a graph $H$ such that $V(H) \subseteq V(G)$ and $E(H) \subseteq E(G)$. A subgraph $H$ is an induced subgraph if two vertices of $V(H)$ are adjacent in $H$ if and only if they are adjacent in $G$. If $U$ is a subset of $V(G)$, then $G \backslash U$ denotes the induced sugraph of $G$ whose vertex set is $V(G) \backslash U$.

A path $P$ of $G$ is a sequence $v_{1}, v_{2}, \ldots, v_{n}$ of distinct vertices such that consecutive vertices are adjacent, and is denoted by $v_{1}-v_{2}-\cdots-v_{n}$. We say that $P$ covers the vertices $v_{1}, \ldots, v_{n}$, and that $v_{j}$ is covered by $P$ for $j=1, \ldots, n$. The vertices $v_{1}$ and $v_{n}$ are the end vertices of $P$. The length of $P$ is defined to be $n-1$ (that is, the length is 1 less than the number of vertices). If each vertex of $G$ belongs to at most one of the paths $P_{1}, \ldots, P_{k}$, then $P_{1}, \ldots, P_{k}$ are disjoint paths of $G$. If $P_{1}=v_{1}-\ldots$ $-v_{s}$ and $P_{2}=v_{s}-\cdots-v_{t}$ are paths whose only common vertex is $v_{s}$, then $P_{1} P_{2}$ denotes the path $v_{1}-\cdots-v_{s}-\cdots-v_{t}$, obtained by concatenating $P_{1}$ and $P_{2}$.

If there is a path between each pair of vertices of $G$, then $G$ is connected; otherwise $G$ is disconnected. A cycle is a connected graph where every vertex has exactly two neighbors. The length of a cycle is the number of its vertices. A cycle of $G$ is a subgraph of $G$ that is a cycle. An acyclic graph is a graph with no cycles. A connected acyclic graph is called a tree, and an acyclic graph is called a forest.

Let $G$ be a connected graph. The distance between two vertices $u$ and $v$ of $G$ is the minimal length of a path from $u$ to $v$. The diameter of a connected graph $G$ is the maximum of the distances between pairs of vertices of $G$, and is denoted by $d(G)$. If $G$ is a tree, then $d(G)$ is the longest length of a path in $G$. The path cover number of $G$ is the minimal number of disjoint paths needed to cover all of the vertices of $G$, and is denoted by $p(G)$.

As is customary, we use graphs to model the combinatorial structure of a matrix. Let $A=\left[a_{i j}\right]$ be an $n$ by $n$ symmetric matrix. The graph $G(A)$ of $A$ consists of the vertices $1,2, \ldots, n$, and the edges $i j$ for which $i \neq j$ and $a_{i j} \neq 0$. Note that $G(A)$ does not depend on the diagonal entries of $A$. The matrix $A$ is acyclic if $G(A)$ is a tree (see [3]). For a given graph $G$ on $n$ vertices, define $S(G)$ to be the set of all $n$ by $n$ real, symmetric matrices with graph $G$, i.e.,

$$
S(G)=\left\{A \in \mathbb{R}^{n \times n} \mid A^{T}=A, G(A)=G\right\} .
$$

For the remainder of this section, matrices are real. Let $\sigma$ be a multi-list of $n$ real numbers. If there exists an $n$ by $n$ symmetric matrix $A$ whose spectrum is $\sigma$, then we say that $\sigma$ is realized by $A$, or $A$ realizes $\sigma$. The spectrum of $S(G)$ for a graph $G$ is the set of all spectra realized by some matrix in $S(G)$. The maximal multiplicity occurring for an eigenvalue of a matrix in $S(G)$ is denoted by MaxMult $(G)$. For a given graph $G$, one can ask to characterize the spectrum of $S(G)$. This characterization problem is known as the Inverse Eigenvalue Problem for the graph $G$, or IEP-G for short. If $G$ is a tree, then we use IEP-T instead of IEP-G.

The IEP-G seems quite difficult. A first step toward resolving the IEP-G for a given graph $G$ is to analyze the possible multiplicities of the eigenvalues in the spectra of matrices in $S(G)$. If the distinct eigenvalues of $A$ are $\lambda_{1}<\lambda_{2}<\cdots<\lambda_{q}$ and their corresponding multiplicities $m_{1}, \ldots, m_{q}$, then $\left\langle m_{1}, m_{2}, \ldots, m_{q}\right\rangle$ is the ordered multiplicity list of the eigenvalues of $A$. For the IEP-T, based on the interplay between the spectral properties of acyclic matrices and the combinatorial properties of trees, 
some significant and intriguing progress has recently been made on (ordered) multiplicity lists (see $[3,8,9,11-14,16]$ ). The significant graphical parameters of trees $T$ considered in those literature are $d(T)$ and $p(T)$. For instance, A. Leal Duarte and C.R. Johnson showed in $[8,14]$ that the minimal number $q(T)$ of distinct eigenvalues over the matrices in $S(T)$ satisfies $q(T) \geq d(T)+1$, and $\operatorname{MaxMult}(T)=p(T)$.

In this paper, we introduce an approach based on the Smith Normal Form to study the spectra of matrices in $S(G)$. In Section 2 we relate the multiplicities of the eigenvalues of an $n$ by $n$ symmetric matrix $A$ to the Smith Normal Form of $x I-A$ where $I$ is the identity matrix. In Section 3 it is proved that an eigenvalue of multiplicity $k+1$ or more of an acyclic matrix $A \in S(T)$ for a tree $T$ must be an eigenvalue of each principal submatrix of $A$ whose graph is obtained from $T$ by deleting $k$ disjoint paths. As an application, we give an example showing that the IEP-T is not equivalent to determining the ordered multiplicity lists of the eigenvalues of matrices in $S(T)$. In Section 4 the known result MaxMult $(T) \leq P(T)$ is demonstrated to be a direct consequence of the Smith Normal Form approach, and a systematic way to compute $p(T)$ for a tree $T$ is given. In Section 5 it is shown that the bound $q(T) \geq d(T)+1$ is also a direct consequence of the Smith Normal Form approach, and that $q(W) \geq \frac{9 d(W)}{8}+\frac{1}{2}$ for an infinite family of trees $W$. In Section 6 it is shown that the Smith Normal Form approach can be applied to graphs that are not trees.

\section{SNF and multiplicities of eigenvalues}

Throughout the remainder of this paper, we let $I$ denote identity matrices. In this section we give some useful results on the Smith Normal Form of matrices over the real polynomial ring $\mathbb{R}[x]$. We refer the reader to $[2,5]$ for the basic facts. In particular, we relate the multiplicities of the eigenvalues of a real, symmetric matrix $A$ to the Smith Normal Form of $x I-A$.

For $p(x), q(x) \in \mathbb{R}[x]$, we write $p(x) \mid q(x)$ if $p(x)$ divides $q(x)$, and $p(x) \nmid q(x)$ if $p(x)$ does not divide $q(x)$. We write $(x-a)^{k} \| q(x)$ when $(x-a)^{k} \mid q(x)$ and $(x-a)^{k+1} \nmid q(x)$. We let $\mathbb{F}$ denote the field of rational functions over $\mathbb{R}$ (that is, $\mathbb{F}$ is the field of quotients of $\mathbb{R}[x])$, and let $(\mathbb{R}[x])^{m \times n}$ denote the set of all $m$ by $n$ matrices over $\mathbb{R}[x]$. The rank of a matrix $M \in(\mathbb{R}[x])^{m \times n}$ is defined to be the rank of $M$ over $\mathbb{F}$. Let $\mathrm{GL}_{n}$ be the set of all invertible matrices of order $n$ over $\mathbb{R}[x]$, i.e.,

$$
\mathrm{GL}_{n}=\left\{P \in(\mathbb{R}[x])^{n \times n} \mid P \text { has an inverse in }(\mathbb{R}[x])^{n \times n}\right\} .
$$

It is a well-known fact that

$$
\mathrm{GL}_{n}=\left\{P \in(\mathbb{R}[x])^{n \times n} \mid \operatorname{det} P \in \mathbb{R} \backslash\{0\}\right\} .
$$

Matrices $M$ and $N$ in $(\mathbb{R}[x])^{m \times n}$ are equivalent over $\mathbb{R}[x]$ if there exist $P \in \mathrm{GL}_{m}$ and $Q \in \mathrm{GL}_{n}$ such that $N=P M Q$. Hence, equivalent matrices have the same rank.

A $k$ by $k$ minor of $M$ is the determinant of a $k$ by $k$ submatrix of $M$. The monic greatest common divisor of all $k$ by $k$ minors of $M$ is the $k t h$ determinantal divisor of $M$ and is denoted by $\Delta_{k}(M)$. Also, define $\Delta_{0}(M)=1$. 
Proposition 1 Let $M, N \in(\mathbb{R}[x])^{m \times n}$. If $M$ and $N$ are equivalent over $\mathbb{R}[x]$, then $\Delta_{k}(M)=\Delta_{k}(N)$ for each $k \in\{1, \ldots, n\}$.

The following fundamental theorem asserts that each square matrix over $\mathbb{R}[x]$ is equivalent to a diagonal matrix over $\mathbb{R}[x]$ of a special form (see $[2,5])$.

Theorem 2 (Smith Normal Form) Let $M \in(\mathbb{R}[x])^{n \times n}$ of rank $r$. Then there exist $P, Q \in \mathrm{GL}_{n}$ and monic polynomials $e_{i}(x)(i=1,2, \ldots, r)$ such that $P M Q=$ $D \oplus O$, where $O$ is the zero matrix of order $n-r, D=\operatorname{diag}\left(e_{1}(x), \ldots, e_{r}(x)\right)$, and $e_{i}(x) \mid e_{i+1}(x)$ for $i=1, \ldots, r-1$. Moreover, $\Delta_{k}(M)=\prod_{j=1}^{k} e_{j}(x)$ and $e_{k}(x)=$ $\frac{\Delta_{k}(M)}{\Delta_{k-1}(M)}$ for each $k \in\{1, \ldots, r\}$.

In the above theorem, $D \oplus O$ is called the Smith Normal Form (SNF) of $M$, and $e_{i}(x)$ is called the ith invariant factor of $M$.

Now assume that $A$ is an $n$ by $n$ real matrix, and let $S$ be the SNF of $x I-A$. The characteristic polynomial of $A$, denoted by $p_{A}(x)$, is $\operatorname{det}(x I-A)=\Delta_{n}(x I-A)$. Since $\operatorname{det}(x I-A)$ is nonzero, the rank of $x I-A$ is $n$. Thus, $S$ is a full rank matrix of the form $\operatorname{diag}\left(e_{1}(x), \ldots, e_{n}(x)\right)$. Since $S$ and $x I-A$ are equivalent over $\mathbb{R}[x]$, Proposition 1 implies that

$$
p_{A}(x)=\prod_{j=1}^{n} e_{j}(x)=\Delta_{n}(S)
$$

Further assume that $A$ is symmetric. Then the spectrum of a symmetric matrix $A$ and the invariant factors of $x I-A$ are closely related. Let $P, Q \in \mathrm{GL}_{n}$ such that $P(x I-A) Q=S$. Since $A$ is symmetric, there exists a real orthogonal matrix $U$ of order $n$ so that $U^{T} A U$ is of the form $D=\operatorname{diag}\left(\lambda_{1}, \ldots, \lambda_{n}\right)$. The diagonal matrix $D$ is called a diagonalization of $A$. Thus, $S=P U(x I-D) U^{T} Q$. Moreover, since (1) implies that $P U, U^{T} Q \in \mathrm{GL}_{n}$, the SNF of $x I-D$ is also $S$. This with Proposition 1 together imply that $\Delta_{i}(x I-A)=\Delta_{i}(x I-D)=\Delta_{i}(S)$ for all $i$.

Henceforth $\Delta_{i}(x)$ denotes $\Delta_{i}(x I-A)$. If $\lambda$ is an eigenvalue of $A$, then $m_{A}(\lambda)$ denotes the algebraic multiplicity of $\lambda$. Let $p(x) \in \mathbb{R}[x]$ and $a \in \mathbb{R}$. If $(x-a) \| p(x)$, then $x-a$ is a linear factor of $p(x)$. By considering $x I-D$, we have the following result on the factors of $\Delta_{i}(x)$ and $e_{i}(x)$.

Theorem 3 Let $A$ be an $n$ by $n$ symmetric matrix whose distinct eigenvalues are $\lambda_{1}, \lambda_{2}, \ldots, \lambda_{q}$ and let $S=\operatorname{diag}\left(e_{1}(x), \ldots, e_{n}(x)\right)$ be the $S N F$ of $x I-A$. Then the following hold.

(a) If $k \leq n-m_{A}\left(\lambda_{j}\right)$, then $\left(x-\lambda_{j}\right) \nmid \Delta_{k}(x)$ and $\left(x-\lambda_{j}\right) \nmid e_{k}(x)$.

(b) If $k>n-m_{A}\left(\lambda_{j}\right)$, then $\left(x-\lambda_{j}\right)^{k-n+m_{A}\left(\lambda_{j}\right)} \| \Delta_{k}(x)$ and $x-\lambda_{j}$ is a linear factor of $e_{k}(x)$.

(c) $e_{n-k}(x)=\prod_{j: m_{A}\left(\lambda_{j}\right)>k}\left(x-\lambda_{j}\right)$. 
Proof Fix $j$, and let $\lambda=\lambda_{j}$, and $m=m_{A}\left(\lambda_{j}\right)$. Let $D$ be a diagonalization of $A$. Without loss of generality, we may assume that

$$
x I-D=\left[\begin{array}{ccc}
x-\mu_{1} & & \\
& \ddots & \\
& & x-\mu_{n-m}
\end{array}\right] \oplus\left[\begin{array}{ccc}
x-\lambda & \\
& \ddots & \\
& & x-\lambda
\end{array}\right]_{m \times m},
$$

where $\mu_{i} \neq \lambda$ for each $i=1, \ldots, n-m$.

(a) Suppose $k \leq n-m$. The determinant of $\operatorname{diag}\left(x-\mu_{1}, \ldots, x-\mu_{k}\right)$ is not divisible by $x-\lambda$. Thus, $(x-\lambda) \nmid \Delta_{k}(x)$. By Theorem $2, e_{k}(x) \mid \Delta_{k}(x)$. Hence, $(x-\lambda) \nmid e_{k}(x)$. (b) Suppose $k>n-m$, and let $M$ be a $k$ by $k$ submatrix of $x I-D$. If $M$ is not a principal submatrix of $x I-D$, then $M$ has a zero row and hence $\operatorname{det} M=0$. Otherwise, at least $k-(n-m)$ diagonal entries of $M$ are $x-\lambda$. Thus, $(x-\lambda)^{k-(n-m)} \mid \Delta_{k}(x)$. Note that $x I-D$ has a $k$ by $k$ minor equal to $\operatorname{det}\left[\operatorname{diag}\left(x-\mu_{1}, \ldots, x-\mu_{n-m}, x-\right.\right.$ $\lambda, \ldots, x-\lambda)]$. Thus $(x-\lambda)^{k-(n-m)+1} \nmid \Delta_{k}(x)$. Hence,

$$
(x-\lambda)^{k-(n-m)} \| \Delta_{k}(x) .
$$

By Theorem 2, $e_{k}(x)=\frac{\Delta_{k}(x)}{\Delta_{k-1}(x)}$. By (3), $\Delta_{k}(x)$ has exactly $k-n+m$ factors equal to $x-\lambda$ and $\Delta_{k-1}(x)$ has exactly $(k-n+m)-1$ factors equal to $x-\lambda$. Thus, $(x-\lambda) \| e_{k}(x)$, and (b) holds.

(c) By (2), $e_{n-k}(x)$ is a product of linear factors from $\left\{x-\lambda_{1}, \ldots, x-\lambda_{q}\right\}$, and by (a) and (b), the factors are distinct, and $x-\lambda_{j}$ is a factor of $e_{n-k}(x)$ if and only if $m_{A}\left(\lambda_{j}\right)>k$. Thus (c) holds.

Useful, immediate consequences of Theorem 3 are the following:

Corollary 4 Let $A$ be an $n$ by $n$ symmetric matrix, and $S=\operatorname{diag}\left(e_{1}(x), \ldots, e_{n}(x)\right)$ be the SNF of $x I-A$. Suppose that $\lambda$ is an eigenvalue of $A$. Then

(a) $m_{A}(\lambda) \geq k$ if and only if $(x-\lambda) \mid e_{n-k+1}(x)$

(b) $m_{A}(\lambda)=k$ if and only if $(x-\lambda) \mid e_{n-k+1}(x)$ but $(x-\lambda) \nmid e_{n-k}(x)$.

Theorem 3(c) implies

$\operatorname{deg}\left(e_{n-k}(x)\right)$ is the number of eigenvalues of $A$ with multiplicity $k+1$ or more.

The minimal polynomial of a square matrix $A$ is the unique, monic polynomial $p(x)$ of smallest degree such that $p(A)=O$. Taking $k=0$ in Theorem 3(c), we see that $e_{n}=\left(x-\lambda_{1}\right)\left(x-\lambda_{2}\right) \cdots\left(x-\lambda_{q}\right)$, which is known to be the minimal polynomial of symmetric matrix $A$. Thus, $\operatorname{deg}\left(e_{n}(x)\right)$ equals the number $q(A)$ of distinct eigenvalues of $A$, and by Theorem 2, we have the following:

Corollary 5 Let $A$ be an $n$ by $n$ symmetric matrix, and $S=\operatorname{diag}\left(e_{1}(x), \ldots, e_{n}(x)\right)$ be the SNF of $x I-A$. Then $e_{n}(x)$ is the minimal polynomial of $A$, and

$$
q(A)=n-\operatorname{deg}\left(\Delta_{n-1}(x)\right) .
$$


Corollary 5 allows one to obtain a lower bound on $q(A)$ from an upper bound on $\operatorname{deg}\left(\Delta_{n-1}(x)\right)$.

\section{Eigenvalues of principal submatrices of an acyclic matrix}

In this section we associate a digraph on $n$ vertices with an $n$ by $n$ matrix $M$, and describe certain minors of $M$ in terms of the structure of the digraph associated with $M$. We use this description to show that an eigenvalue of $A \in S(T)$ with multiplicity $k+1$ or more is an eigenvalue of each principal submatrix of $A$ whose rows and columns correspond to the vertices not covered by a set of $k$ disjoint paths. As an application, we provide an example showing that the IEP-T is not equivalent to determining the ordered multiplicity lists of the eigenvalues of matrices in $S(T)$ (see also [1]).

Let $M=\left[m_{i j}\right]$ be an $n$ by $n$ matrix. The digraph $D(M)$ of $M$ consists of the vertices $1,2, \ldots, n$, and $\operatorname{arcs}(i, j)$ from vertex $i$ to vertex $j$ if and only if $m_{i j} \neq 0$. The underlying graph of the digraph $D(M)$ is the graph obtained by treating each $\operatorname{arc}(i, j)(i \neq j)$ of $D(M)$ as the edge $i j$, and ignoring the loops. A directed walk in $D(M)$ is a sequence of vertices $\left(v_{1}, v_{2}, \ldots, v_{\ell}\right)$, such that $\left(v_{i}, v_{i+1}\right)$ is an arc for each $i=1, \ldots, \ell-1$, and $v_{1}$ is the initial vertex and $v_{\ell}$ is the terminal vertex of the directed walk. The directed walk $\left(v_{1}, v_{2}, \ldots, v_{\ell}\right)$ covers the vertices $v_{1}, v_{2}, \ldots, v_{\ell}$, and has length $\ell-1$. If no vertex of a directed walk is repeated, then the directed walk is a directed path.

If digraph $D(M)$ has a unique directed path from vertex $i$ to vertex $j$, then the unique directed path is denoted by $P_{i \rightarrow j}$. If the underlying graph of $D(M)$ has a unique path connecting vertex $i$ and vertex $j$, then the path is denoted by $P_{i-j}$.

The weight of the arc $(i, j)$ of $D(M)$ is $m_{i j}$. The weight of a directed walk $\beta$ of $D(M)$ is the product of the weights of its arcs, and is denoted by $\operatorname{wt}(\beta)$.

If $\alpha$ and $\beta$ are subsets of $\{1, \ldots, n\}$, then we denote the submatrix of $M$ obtained by removing (resp. retaining) rows indexed by $\alpha$ and columns indexed by $\beta$ by $M(\alpha, \beta)$ (resp. $M[\alpha, \beta]$ ). When $\alpha=\beta$, we use $M(\alpha)$ and $M[\alpha]$, respectively.

The following result ([15, Theorem 9]) will be a useful tool throughout the remainder of the paper.

Theorem 6 Let $M$ be an $n \times n$ matrix. Then, for $i, j \in\{1,2, \ldots, n\}$ with $i \neq j$,

$$
\operatorname{det} M(\{j\},\{i\})=(-1)^{i+j} \sum_{P}(-1)^{\ell(P)} \mathrm{wt}(P) \operatorname{det} M(V(P))
$$

where the sum is taken over all directed paths $P$ in $D(M)$ from $i$ to $j$, and $\ell(P)$ is the length of $P$.

Note that if $M$ is acyclic, then for each pair of distinct vertices $i$ and $j$ there is a unique directed path from $i$ to $j$ in $D(M)$, and hence

$$
\operatorname{det} M(\{j\},\{i\})=(-1)^{i+j+\ell\left(P_{i \rightarrow j}\right)} \mathrm{wt}\left(P_{i \rightarrow j}\right) \operatorname{det} M\left(V\left(P_{i-j}\right)\right) .
$$


Corollary 7 Let $M$ be an $n$ by $n$ matrix having digraph $D$ with underlying graph $G$, and let $i_{1}, i_{2}, \ldots, i_{k}, j_{1}, j_{2}, \ldots, j_{k} \in\{1,2, \ldots, n\}$ be distinct. Suppose that for each $r=1,2, \ldots, k$, there is a directed path $P_{i_{r} \rightarrow j_{r}}$ from $i_{r}$ to $j_{r}$ in $D$ with underlying path $P_{i_{r}-j_{r}}$ in $G$ such that $P_{i_{r}-j_{r}}$ is the unique path connecting $i_{r}$ and $j_{r}$ in $G$ and paths $P_{i_{1}-j_{1}}, \ldots, P_{i_{k}-j_{k}}$ are disjoint. Then

$$
\begin{aligned}
\operatorname{det} & M\left(\left\{j_{1}, \ldots, j_{k}\right\},\left\{i_{1}, \ldots, i_{k}\right\}\right) \\
= \pm & \prod_{r=1}^{k} \operatorname{wt}\left(P_{i_{r} \rightarrow j_{r}}\right) \cdot \operatorname{det} M\left(V\left(P_{i_{1}-j_{1}}\right) \cup \cdots \cup V\left(P_{i_{k}-j_{k}}\right)\right) .
\end{aligned}
$$

Proof The proof is by induction on $k$. The statement is vacuously true for $k=0$, and the case $k=1$ is true by Theorem 6 . Let $k \geq 2$ and assume that the statement is true for $k-1$.

Let $B$ be the matrix obtained from $M$ by interchanging columns $j_{r}$ and $i_{r}$ for each $r=1, \ldots, k-1$. Then, since path $P_{i_{k}-j_{k}}$ is the unique path in $G$ connecting $i_{k}$ and $j_{k}$ that is disjoint from paths $P_{i_{1}-j_{1}}, \ldots, P_{i_{k-1}-j_{k-1}}$, the directed path $P_{i_{k} \rightarrow j_{k}}$ is still the unique directed path from $i_{k}$ to $j_{k}$ in the digraph $D\left(B\left(\left\{j_{1}, \ldots, j_{k-1}\right\}\right)\right)$ with the underlying path $P_{i_{k}-j_{k}}$. Hence, it follows that

$$
\begin{aligned}
\operatorname{det} & M\left(\left\{j_{1}, \ldots, j_{k}\right\},\left\{i_{1}, \ldots, i_{k}\right\}\right) \\
= & \pm \operatorname{det}\left[B\left(\left\{j_{1}, \ldots, j_{k-1}\right\}\right)\right]\left(\left\{j_{k}\right\},\left\{i_{k}\right\}\right) \\
= & \pm \operatorname{wt}\left(P_{i_{k} \rightarrow j_{k}}\right) \operatorname{det}\left[B\left(\left\{j_{1}, \ldots, j_{k-1}\right\}\right)\right]\left(V\left(P_{i_{k}-j_{k}}\right)\right) \text { by Theorem } 6 \\
= & \pm \operatorname{wt}\left(P_{i_{k} \rightarrow j_{k}}\right) \operatorname{det}\left[B\left(V\left(P_{i_{k}-j_{k}}\right)\right)\right]\left(\left\{j_{1}, \ldots, j_{k-1}\right\}\right) \\
= & \pm \operatorname{wt}\left(P_{i_{k} \rightarrow j_{k}}\right) \operatorname{det}\left[M\left(V\left(P_{i_{k}-j_{k}}\right)\right)\right]\left(\left\{j_{1}, \ldots, j_{k-1}\right\},\left\{i_{1}, \ldots, i_{k-1}\right\}\right) .
\end{aligned}
$$

Since matrix $M\left(V\left(P_{i_{k}-j_{k}}\right)\right)$ with indices $i_{1}, \ldots, i_{k-1}, j_{1}, \ldots, j_{k-1}$ satisfies the condition in the statement, the result follows by the inductive hypothesis.

Theorem 8 Let $A \in S(T)$ where $T$ is a tree on $n$ vertices and let $S=\operatorname{diag}\left(e_{1}(x), \ldots\right.$, $\left.e_{n}(x)\right)$ be the SNF of $x I-A$. If $P_{i_{1}-j_{1}}, \ldots, P_{i_{k}-j_{k}}$ are disjoint paths in $T$ covering $n-t$ vertices of $T$, then

$$
\Delta_{n-k}(x) \mid \operatorname{det}(x I-A)\left(V\left(P_{i_{1}-j_{1}}\right) \cup \cdots \cup V\left(P_{i_{k}-j_{k}}\right)\right) \text { and } \operatorname{deg}\left(\Delta_{n-k}(x)\right) \leq t .
$$

Furthermore, if $\lambda$ is an eigenvalue of $A$ with $m_{A}(\lambda) \geq k+1$, then $\lambda$ is an eigenvalue of $A\left(V\left(P_{i_{1}-j_{1}}\right) \cup \cdots \cup V\left(P_{i_{k}-j_{k}}\right)\right)$ with multiplicity $m_{A}(\lambda)-k$ or more.

Proof For each $s=1, \ldots, k$, let $P_{i_{s} \rightarrow j_{s}}$ be the directed path from $i_{s}$ to $j_{s}$ in digraph $D(x I-A)$ with underlying graph $P_{i_{s}-j_{s}}$. By Corollary 7 ,

$$
\begin{aligned}
& \operatorname{det}(x I-A)\left(\left\{j_{1}, \ldots, j_{k}\right\},\left\{i_{1}, \ldots, i_{k}\right\}\right) \\
& \quad= \pm \prod_{s=1}^{k} \mathrm{wt}\left(P_{i_{s} \rightarrow j_{s}}\right) \cdot \operatorname{det}(x I-A)\left(V\left(P_{i_{1}-j_{1}}\right) \cup \cdots \cup V\left(P_{i_{k}-j_{k}}\right)\right) .
\end{aligned}
$$


Fig. 1

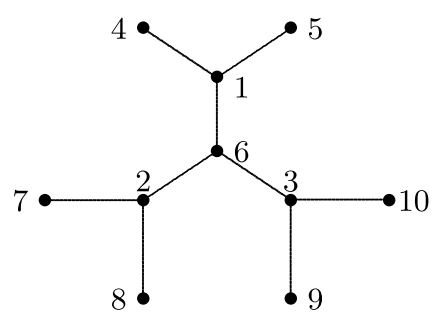

Since each $\operatorname{wt}\left(P_{i_{s} \rightarrow j_{s}}\right)$ is a nonzero constant and $(x I-A)\left(\left\{j_{1}, \ldots, j_{k}\right\},\left\{i_{1}, \ldots, i_{k}\right\}\right)$ is an $n-k$ by $n-k$ submatrix of $x I-A$,

$$
\Delta_{n-k}(x) \mid \operatorname{det}(x I-A)\left(V\left(P_{i_{1}-j_{1}}\right) \cup \cdots \cup V\left(P_{i_{k}-j_{k}}\right)\right) .
$$

Suppose that $\lambda$ is an eigenvalue of $A$ with $m_{A}(\lambda) \geq k+1$. By Theorem 3 , the multiplicity of $(x-\lambda)$ as a factor of $\Delta_{n-k}(x)$ is $m_{A}(\lambda)-k$. Therefore, by (5), $(x-\lambda)^{m_{A}(\lambda)-k} \mid \operatorname{det}(x I-A)\left(V\left(P_{i_{1}-j_{1}}\right) \cup \cdots \cup V\left(P_{i_{k}-j_{k}}\right)\right)$. This implies that $\lambda$ is an eigenvalue of $A\left(V\left(P_{i_{1}-j_{1}}\right) \cup \cdots \cup V\left(P_{i_{k}-j_{k}}\right)\right)$ with multiplicity $m_{A}(\lambda)-k$ or more.

Note that the degree of polynomial $\operatorname{det}(x I-A)\left(V\left(P_{i_{1}-j_{1}}\right) \cup \cdots \cup V\left(P_{i_{k}-j_{k}}\right)\right)$ is $t$. Thus, by (5),

$$
\operatorname{deg}\left(\Delta_{n-k}(x)\right) \leq t
$$

If $\operatorname{deg}\left(\Delta_{n-k}(x)\right) \leq t$, then Theorem 2 implies that $\operatorname{deg}\left(e_{n-k}(x)\right) \leq t$. Hence, Corollary 4 implies the following.

Corollary 9 Let $A \in S(T)$, where $T$ is a tree on $n$ vertices. If $k$ disjoint paths of $T$ cover $n-t$ vertices of $T$, then there are at most $t$ eigenvalues of $A$ with multiplicity $k+1$ or more.

It was conjectured in [11] that the IEP-T for a tree $T$ is equivalent to determining the ordered multiplicity lists of the eigenvalues of matrices in $S(T)$, i.e., each multilist of real numbers of the eigenvalues of a matrix in $S(T)$ is the spectrum of a matrix in $S(T)$. Indeed, it was shown in [11] that for some classes of trees, these two problems are equivalent. A counterexample to the conjecture was given in [1]. We give a counterexample on fewer vertices with a simple argument. This counterexample was motivated by that in Figure 2.2 of [1].

Example 10 Consider the tree $T$ illustrated in Figure 1. We will show that an ordered multiplicity list of the eigenvalues of a matrix in $S(T)$ requires the eigenvalues having the ordered multiplicity list to satisfy a certain algebraic condition.

It can be verified that the eigenvalues of the $(0,1)$-matrix $A$ in $S(T)$ with trace 0 are $-\sqrt{5},-\sqrt{2}, 0, \sqrt{2}, \sqrt{5}$, and the ordered multiplicity list of the eigenvalues of $A$ is $\langle 1,2,4,2,1\rangle$.

Suppose that

$$
\sigma=\left(\lambda_{1}, \lambda_{2}, \lambda_{2}, \lambda_{3}, \lambda_{3}, \lambda_{3}, \lambda_{3}, \lambda_{4}, \lambda_{4}, \lambda_{5}\right)
$$


is realized by a matrix $B$ in $S(T)$ as its spectrum where $\lambda_{1}<\lambda_{2}<\lambda_{3}<\lambda_{4}<\lambda_{5}$. The disjoint paths $4-1-5,7-2-8$ and $10-3-9$ cover all the vertices except vertex 6 . Since $m_{B}\left(\lambda_{3}\right)=4$, Corollary 8 implies that $\lambda_{3}$ is the eigenvalue of $B[\{6\}]$, i.e., $B[\{6\}]=\lambda_{3}$.

Next, since the three disjoint paths $4-1-6-2-7,10-3-9$, and 8 cover all the vertices except vertex 5 , Theorem 8 implies that $\lambda_{3}$ is the eigenvalue of $B[\{5\}]$, i.e., $B[\{5\}]=\lambda_{3}$. Similarly, $\lambda_{3}$ is the eigenvalue of $B[\{i\}]$, i.e., $B[\{i\}]=\lambda_{3}$ for each $i=4,5,7,8,9,10$.

The three disjoint paths $7-2-6-3-10,8$ and 9 cover seven vertices. Thus, by Theorem $8, \lambda_{3}$ is an eigenvalue of $B[\{4,1,5\}]$. Similarly, $\lambda_{3}$ is an eigenvalue of $B[\{10,3,9\}]$ and $B[\{7,2,8\}]$.

Finally, we show that both eigenvalues of $B$ with multiplicity 2 are eigenvalues of $B[\{4,1,5\}], B[\{7,2,8\}]$ and $B[\{10,3,9\}]$. We consider only the case for $B[\{4,1,5\}]$. Since the single path 7-2-6-3-10 covers five vertices, and $m_{B}\left(\lambda_{2}\right)=m_{B}\left(\lambda_{4}\right)=$ 2, Theorem 8 implies that $\lambda_{2}$ and $\lambda_{4}$ are eigenvalues of $B[\{4,1,5,8,9\}]$. However, $B[\{4,1,5,8,9\}]=B[\{4,1,5\}] \oplus B[\{8\}] \oplus B[\{9\}]$. Since $B[\{8\}]=B[\{9\}]=\lambda_{3}, \lambda_{2}$ and $\lambda_{4}$ are eigenvalues of $B[\{4,1,5\}]$. The other cases can be shown by choosing the paths $4-1-6-2-7$ and $4-1-6-3-10$, respectively.

So far, we have shown that $\sigma(B[\{6\}])=\lambda_{3}$, and $\sigma(B[\{4,1,5\}])=\sigma(B[\{7,2,8\}])$ $=\sigma(B[\{10,3,9\}])=\left(\lambda_{2}, \lambda_{3}, \lambda_{4}\right)$. Now, we consider the trace of $B$. The trace of $B$ is equal to the sum of the traces of $B[\{6\}], B[\{4,1,5\}], B[\{7,2,8\}]$ and $B[\{10,3,9\}]$. Since the trace is equal to the sum of all the eigenvalues, we have

$$
\lambda_{1}+2 \lambda_{2}+4 \lambda_{3}+2 \lambda_{4}+\lambda_{5}=\lambda_{3}+3\left(\lambda_{2}+\lambda_{3}+\lambda_{4}\right)
$$

and hence,

$$
\lambda_{1}+\lambda_{5}=\lambda_{2}+\lambda_{4} .
$$

Therefore, if the ordered multiplicity list $\langle 1,2,4,2,1\rangle$ is realized by a matrix $B$ in $S(T)$, then $\sigma(B)=\left(\lambda_{1}, \lambda_{2}, \lambda_{2}, \lambda_{3}, \lambda_{3}, \lambda_{3}, \lambda_{3}, \lambda_{4}, \lambda_{4}, \lambda_{5}\right)$ must satisfy $\lambda_{1}+\lambda_{5}=$ $\lambda_{2}+\lambda_{4}$. For instance, $\sigma=(2,3,3,5,5,5,5,7,7,10)$ with the ordered multiplicity list $\langle 1,2,4,2,1\rangle$ cannot be realized by any matrix in $S(T)$.

\section{Maximal multiplicity and $p(T)$}

Let $T$ be a tree on $n$ vertices. Recall that $\operatorname{MaxMult}(T)$ is the maximal multiplicity occurring for an eigenvalue among the matrices in $S(T)$, and the path cover number $p(T)$ of $T$ is the minimal number of disjoint paths that cover $T$.

Let $A \in S(T)$. If $k$ disjoint paths in $T$ cover all the vertices of $T$, then Corollary 9 implies that no eigenvalue of $A$ has multiplicity more than $k$. Since $A$ is an arbitrary matrix in $S(T)$, it follows that $\operatorname{MaxMult}(T) \leq p(T)$. In [8], it was shown that in fact equality holds.

Theorem 11 If $T$ is a tree, then

$$
\operatorname{MaxMult}(T)=p(T)
$$


For the remainder of this section, we describe a systematic way of computing $p(T)$. This method will be used repeatedly in the following sections. We first show the existence of a specific path for a given tree (see also [16, Lemma 3.1]).

Proposition 12 Let $T$ be a tree on $n$ vertices. Then there exists a path in $T$ such that the end vertices of the path are pendant vertices of $T$, and at most one vertex of the path has degree 3 or more in $T$.

Proof The proof is by induction on $n$. The result is clearly true if $n \leq 2$. Assume that $n \geq 3$ and proceed by induction.

If $T$ has diameter 2 , then any path of length 2 works. Assume that the diameter of $T$ is at least 3, and let $P$ be a path in $T, u-v-a_{1}-\cdots-a_{k-1}-a_{k}$, whose length is the diameter of $T$. Since $u$ is a pendant vertex of $T, T \backslash\{u\}$ is also a tree. By the inductive hypothesis, there exists a path $P^{\prime}$ of $T \backslash\{u\}$ satisfying the given condition.

If the path $P^{\prime}$ in $T \backslash\{u\}$ does not contain $v$, then $P^{\prime}$ is also a path in $T$ satisfying the given condition. If $P^{\prime}$ contains $v$ as an end vertex, then the path $P_{u-v} P^{\prime}$ is a path in $T$ satisfying the given condition. Otherwise $P^{\prime}$ contains $v$ and $v$ is not an end vertex of $P^{\prime}$. Thus there exists a neighbor $w$ of $v$ in $T \backslash\{u\}$ such that $w \neq a_{1}$. Suppose that $w$ is not a pendant vertex of $T$. Then there exists a neighbor $y$ of $w$ other than $v$. Since $T$ is a tree, $y \notin\left\{a_{2}, \ldots, a_{k}\right\}$ and hence, $y-w-v-a_{1}-\ldots-a_{k-1}-a_{k}$ is a path in $T$, whose length is longer than $P$, which is a contradiction. Therefore, $w$ is a pendant vertex of $T$, and the path $u-v-w$ in $T$ satisfies the given condition.

If $T$ is a path, then a path in $T$ satisfying the conditions in Proposition 12 is $T$ itself. Otherwise, there exists a path $P$ in $T$ such that the end vertices of $P$ are pendant vertices of $T$, and exactly one vertex of $P$ has degree 3 or more in $T$. Next, we show that for such a path $P, p(T \backslash V(P))=p(T)-1$.

Proposition 13 Let $T$ be a tree that is not a path. Suppose that $P$ is a path in $T$ such that $P$ 's end vertices are pendant vertices of $T$ and $P$ has exactly one vertex $v$ of degree 3 or more in $T$. Then

$$
p(T)=p(T \backslash V(P))+1 \text {. }
$$

Proof Note that each path cover of $T \backslash V(P)$ can be extended to a path cover of $T$ by including the path $P$. Hence $p(T) \leq p(T \backslash V(P))+1$.

It is shown next that $p(T) \geq p(T \backslash V(P))+1$. Let $p$ denote $p(T)$ and $C=\left\{P_{i}\right\}_{i=1}^{p}$ be a set of $p$ disjoint paths in $T$ covering all of the vertices of $T$. If $P \in C$, then, since $C \backslash\{P\}$ covers all of the vertices of $T \backslash V(P), p(T \backslash V(P)) \leq p-1$. Otherwise, $P \notin C$. Then two disjoint paths in $C$, say $\alpha$ and $\beta$, are needed to cover the vertices of $P$. Assume that $\beta$ covers the vertex $v$. Then $\alpha$ covers only the vertices of $P$. Thus, $(C \backslash\{\alpha, \beta\}) \cup\{\beta \backslash V(P)\}$ covers all the vertices of $T \backslash P$. This implies that $p(T \backslash V(P)) \leq p-1$; equivalently, $p \geq p(T \backslash V(P))+1$.

Example 14 By repeated use of Proposition 13, we can efficiently compute the path cover number of the tree $T$ in Figure 2. 
Fig. 2

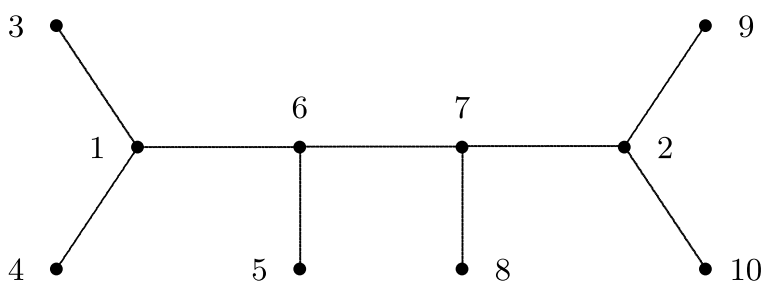

Note that $P_{1}=3-1-4$ and $P_{2}=9-2-10$ satisfy the condition in Proposition 13 for $T$ and $T \backslash V\left(P_{1}\right)$, respectively, and the disjoint paths $P_{1}, P_{2}$ and $P_{3}=5-6-7-8$ cover all of the vertices of $T$. Hence, by Proposition 13, $p(T)=3$. This implies, by Theorem 11, that if a multi-list $\sigma$ of 10 real numbers has an element with multiplicity greater than 3, then $\sigma$ cannot be realized by any matrix in $S(T)$.

\section{Relationship between $d(T)$ and $q(T)$}

In this section, we study the relation between $d(T)$ and $q(T)$.

Let $T$ be a tree on $n$ vertices and $A \in S(T)$. By Corollary 5,

$$
q(A)=n-\operatorname{deg}\left(\Delta_{n-1}(x)\right)
$$

and by Theorem 8 , if a path in $T$ has $\ell$ vertices, then

$$
\operatorname{deg}\left(\Delta_{n-1}(x)\right) \leq n-\ell
$$

Thus, by choosing a path of the longest length, (6) and (7) imply that

$$
q(A) \geq d(T)+1
$$

Since (8) holds for every matrix in $S(T), q(T) \geq d(T)+1$. Thus, the following known theorem (see [14]) follows easily from the Smith Normal Form approach.

Theorem 15 Let $T$ be a tree. Then

$$
q(T) \geq d(T)+1
$$

Next, we provide a class of trees $W$ for which $q(W)$ is much larger than $d(W)+1$. The $(k, \ell)$-whirl $W$ for $k \geq 2$ and $\ell \geq 1$ is the tree on $n=2 k \ell+k+1$ vertices consisting of an axis vertex $v$, vertices $v_{i}$ adjacent to $v$ and pendant paths $\alpha_{1}^{i}, \alpha_{2}^{i}$ of length $\ell$ whose end vertices are adjacent to $v_{i}$ for $i=1,2, \ldots, k$. The $2 k$ pendant paths are called the legs of $W$. Note that $d(W)=2 \ell+2$. If the number of vertices of each leg of $W$ is not specified, we say that $W$ is a $k$-whirl. If the numbers of the vertices of the $2 k$ legs are not necessarily equal, then $W$ is a generalized $k$-whirl. Figure 3 illustrates the $(3, \ell)$-whirl $(\ell \geq 1)$.

Some basic properties of generalized $k$-whirls are the following. 
Fig. 3

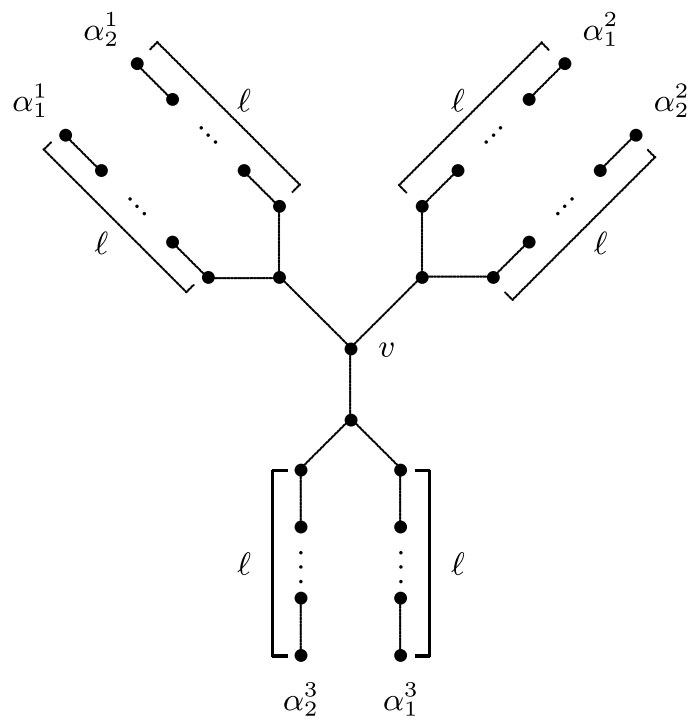

Lemma 16 Suppose that $W$ is a generalized $k$-whirl $(k \geq 2)$ on $n$ vertices with $2 k$ legs $\left\{\alpha_{1}^{i}, \alpha_{2}^{i}\right\}_{i=1}^{k}$ such that each leg has at least one vertex. Let $A \in S(W)$ and let $A^{\prime}$ be the direct sum of $A\left[\alpha_{j}^{i}\right]$ for all $i \in\{1, \ldots, k\}$ and $j \in\{1,2\}$. If $n_{r}$ denotes the number of eigenvalues of $A$ with multiplicity $r$, then the following hold:

(a) $n_{k+1} \leq 1$ and $n_{j}=0$ for $j \geq k+2$;

(b) If $\lambda \in \sigma(A)$ and $m_{A}(\lambda)=k+1$, then $\lambda$ is a simple eigenvalue of $A\left[\alpha_{j}^{i}\right]$ for all $i \in\{1, \ldots, k\}$ and $j \in\{1,2\}$, and $m_{A^{\prime}}(\lambda)=2 k$;

(c) If $\mu \in \sigma(A)$ and $m_{A}(\mu)=k$, then, for all $i \neq s, j$ and $t, \mu$ is a simple eigenvalue of at least one of $A\left[\alpha_{j}^{i}\right], A\left[\alpha_{t}^{s}\right]$, and $m_{A^{\prime}}(\mu) \geq 2 k-2 ;$ and

(d) $(2 k-2) n_{k}+(2 k) n_{k+1} \leq n-(k+1)$.

Proof (a) Let $P_{i}=\alpha_{1}^{i} v_{i} \alpha_{2}^{i}$ for $i=1, \ldots, k$. By applying Proposition 13 to the paths $P_{1}, \ldots, P_{k}$, we conclude that $p(W)=k+1$. Hence, Theorem 11 implies that there is no eigenvalue of $A$ with multiplicity $k+2$ or more, that is, $n_{j}=0$ for $j \geq k+2$. Furthermore, since the $k$ disjoint paths $P_{1}, \ldots, P_{k}$ in $W$ cover all of the vertices of $W$ except $v$, Corollary 9 implies that there exists at most one eigenvalue of $A$ with multiplicity $k+1$, that is, $n_{k+1} \leq 1$. This proves (a).

(b) Suppose that $\lambda \in \sigma(A)$ and $m_{A}(\lambda)=k+1$. Consider the following $k$ disjoint paths in $W: Q_{1}=\alpha_{2}^{1}\left(v_{1}-v-v_{k}\right) \alpha_{2}^{k}, Q_{i}=\alpha_{1}^{i} v_{i} \alpha_{2}^{i}$ for $i=2, \ldots, k-1$ and $Q_{k}=\alpha_{1}^{k}$. Then $W \backslash\left(V\left(Q_{1}\right) \cup \cdots \cup V\left(Q_{k}\right)\right)$ is $\alpha_{1}^{1}$ and hence, Theorem 8 implies that $\lambda \in \sigma\left(A\left[\alpha_{1}^{1}\right]\right)$. By Theorem $11, \lambda$ is a simple eigenvalue of $A\left[\alpha_{1}^{1}\right]$. Similarly, $\lambda$ is a simple eigenvalue of $A\left[\alpha_{2}^{1}\right]$ and $A\left[\alpha_{j}^{i}\right]$ for each $i=2, \ldots, k$ and $j=1,2$. Therefore, $m_{A^{\prime}}(\lambda)=2 k$, and (b) holds.

(c) Suppose that $\mu \in \sigma(A)$ and $m_{A}(\mu)=k$. Let $R_{1}=\alpha_{2}^{1}\left(v_{1}-v-v_{k}\right) \alpha_{2}^{k}$, and $R_{i}=\alpha_{1}^{i} v_{i} \alpha_{2}^{i}$ for $i=2, \ldots, k-1$. Then $A\left[W \backslash\left(V\left(R_{1}\right) \cup \cdots \cup V\left(R_{k-1}\right)\right)\right]$ is, up to permutation similarity, equal to $A\left[\alpha_{1}^{1}\right] \oplus A\left[\alpha_{1}^{k}\right]$. Thus, by Theorem $8, \mu$ is an eigenvalue 
of $A\left[\alpha_{1}^{1}\right] \oplus A\left[\alpha_{1}^{k}\right]$ and hence, by Theorem $11, \mu$ is a simple eigenvalue of at least one of $A\left[\alpha_{1}^{1}\right], A\left[\alpha_{1}^{k}\right]$. Similarly, $\mu$ is a simple eigenvalue of at least one of $A\left[\alpha_{j}^{i}\right]$, $A\left[\alpha_{t}^{s}\right]$ for all $i, s \in\{1, \ldots, k\}, i \neq s$ and $j, t \in\{1,2\}$. If $\mu$ is a simple eigenvalue of at most $2 k-3$ of the $2 k$ matrices in $\left\{A\left[\alpha_{1}^{i}\right], A\left[\alpha_{2}^{i}\right]\right\}_{i=1}^{k}$, then there is a pair of $A\left[\alpha_{j}^{i}\right], A\left[\alpha_{t}^{s}\right]$ for $i \neq s$ such that none of $A\left[\alpha_{j}^{i}\right], A\left[\alpha_{t}^{s}\right]$ has $\mu$ as an eigenvalue. Thus, $\mu$ is a simple eigenvalue of at least $2 k-2$ matrices in $\left\{A\left[\alpha_{1}^{i}\right], A\left[\alpha_{2}^{i}\right]\right\}_{i=1}^{k}$. Therefore, $m_{A^{\prime}}(\mu) \geq 2 k-2$, and (c) holds.

(d) The order of $A^{\prime}$ is $n-(k+1)$. Since the number of eigenvalues of $A^{\prime}$ cannot exceed the order of $A^{\prime},(2 k-2) n_{k}+(2 k) n_{k+1} \leq n-(k+1)$.

Theorem 17 Let $W$ be the $(k, \ell)$-whirl with $k \geq 3$ and $\ell \geq 2$. Then

$$
q(W) \geq d(W)+1+\frac{(k-2)(\ell-1)}{(k-1)^{2}}>d(W)+1 .
$$

In particular, if $W$ is a $(3, \ell)$-whirl, then

$$
q(W) \geq 9 d(W) / 8+1 / 2 .
$$

Proof Let $A \in S(W)$ and let $n_{j}$ be the number of eigenvalues of $A$ with multiplicity $j$. By Lemma 16(a),

$$
n=2 k \ell+k+1=\sum_{i=1}^{k+1} i \cdot n_{i} .
$$

Moreover,

$$
\begin{aligned}
2 k \ell+k+1 & =\sum_{i=1}^{k+1} i \cdot n_{i} \\
& =n_{1}+2 n_{2}+\cdots+k n_{k}+(k+1) n_{k+1} \\
& =2+\left(n_{1}-2+2 n_{2}+\cdots+(k-1) n_{k}+(k-1) n_{k+1}\right)+n_{k}+2 n_{k+1} .
\end{aligned}
$$

By [10, Corollary 7], it follows that $n_{1} \geq 2$. Thus,

$$
\begin{aligned}
2 k \ell+k+1 & \leq 2+(k-1)\left(n_{1}-2+n_{2}+\cdots+n_{k}+n_{k+1}\right)+n_{k}+2 n_{k+1} \\
& =2+(k-1)(q(A)-2)+n_{k}+2 n_{k+1} .
\end{aligned}
$$

Next, since $n=2 k \ell+k+1$, Lemma 16(d) implies

$$
(2 k-2) n_{k}+(2 k) n_{k+1} \leq 2 k \ell .
$$

Thus, by solving (10) for $n_{k}$, we have

$$
n_{k} \leq \frac{k \ell-k n_{k+1}}{k-1} .
$$


Furthermore, by (9),

$$
\begin{aligned}
2 k \ell+k+1 & \leq 2+(k-1)(q(A)-2)+\frac{k \ell-k n_{k+1}}{k-1}+2 n_{k+1} \\
& =(k-1) q(A)-2 k+4+\frac{k \ell+(k-2) n_{k+1}}{k-1} .
\end{aligned}
$$

By Lemma 16(a), $n_{k+1} \leq 1$ and hence,

$$
2 k \ell+3 k-3 \leq(k-1) q(A)+\frac{k \ell+k-2}{k-1} .
$$

By solving (11) for $q(A)$, we have

$$
\begin{aligned}
q(A) & \geq \frac{(2 k \ell+3 k-3)(k-1)-(k \ell+k-2)}{(k-1)^{2}} \\
& =\frac{2 k^{2} \ell+3 k^{2}-3 k \ell-7 k+5}{(k-1)^{2}} \\
& =2 \ell+3+\frac{(k-2)(\ell-1)}{(k-1)^{2}} .
\end{aligned}
$$

Since $d(W)=2 \ell+2$, we have

$$
q(A) \geq d(W)+1+\frac{(k-2)(\ell-1)}{(k-1)^{2}} .
$$

Since $A$ is an arbitrary matrix in $S(W),(12)$ implies that

$$
q(W) \geq d(W)+1+\frac{(k-2)(\ell-1)}{(k-1)^{2}}>d(W)+1
$$

for $k \geq 3$ and $\ell \geq 2$.

For $k=3$, using $d(W)=2 \ell+2$, this simplifies to $q(W) \geq 9 d(W) / 8+2$, as claimed.

\section{$6 q(G)$ for a class of connected graphs $G$}

In the previous sections the Smith Normal Form approach is shown to be an efficient tool for acyclic graphs. In this section, we illustrate the promise of the Smith Normal Form approach for more than just acyclic graphs by finding a lower bound on $q(G)$ for an infinite class of connected graphs $G$ described in Figure 4.

Theorem 18 Let $G$ be a connected graph on $n$ vertices that consists of a connected subgraph $H$ on $m$ vertices containing vertices $v_{1}, v_{2}, v_{3}$ such that for all $r, s$, and $t$ with $\{r, s, t\}=\{1,2,3\}$, there exists a unique shortest path from $v_{s}$ to $v_{t}$ that does not 
Fig. 4

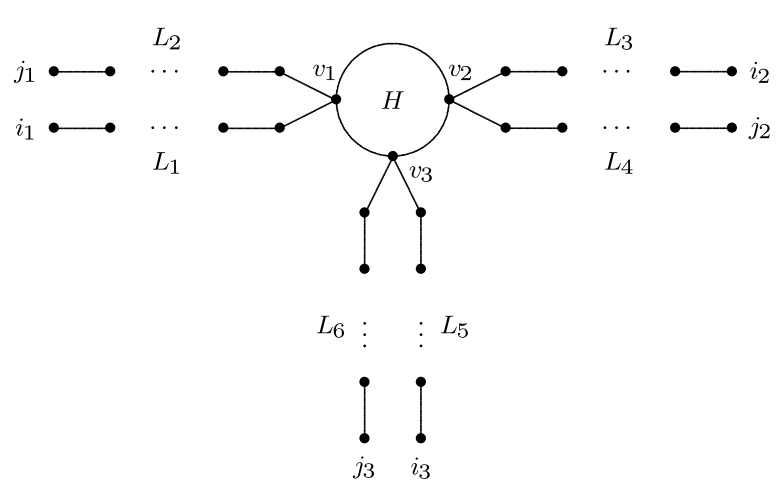

pass $v_{r}$; and 6 legs $L_{1}, \ldots, L_{6}$ on $\ell$ vertices such that end vertices of $L_{1}, L_{2}$ (resp. $L_{3}, L_{4}$ and $\left.L_{5}, L_{6}\right)$ are adjacent to vertex $v_{1}$ (resp. $v_{2}$ and $v_{3}$ ). Then

$$
q(G) \geq \frac{9 \ell}{4}-2 m+\frac{15}{2} .
$$

Proof Let $A \in S(G)$ and $\lambda_{1}, \ldots, \lambda_{q}$ be the distinct eigenvalues of $A$ and let $n_{j}$ be the number of eigenvalues of $A$ with multiplicity $j$. We use $i_{u}$ and $j_{v}$ to denote the pendant vertices of the 6 legs in $G$ (see Figure 4).

Consider $M=x I-A$. We compute the determinant of an $(n-3)$ by $(n-3)$ submatrix of $M$, and find an upper bound on $\sum_{\lambda_{j}: m_{A}\left(\lambda_{j}\right) \geq 4}\left(m_{A}\left(\lambda_{j}\right)-2\right)$. In $G$ there is a unique path $P_{i_{s}-j_{s}}$ from $i_{s}$ to $j_{s}$ and a unique directed path $P_{i_{s} \rightarrow j_{s}}$ from $i_{s}$ to $j_{s}$ for $s=1,2,3$. Since $P_{i_{1}-j_{1}}, P_{i_{2}-j_{2}}$ and $P_{i_{3}-j_{3}}$ are disjoint, Corollary 7 implies that

$$
\operatorname{det} M\left(\left\{j_{1}, j_{2}, j_{3}\right\},\left\{i_{1}, i_{2}, i_{3}\right\}\right)= \pm \prod_{s=1}^{3} \operatorname{wt}\left(P_{i_{s} \rightarrow j_{s}}\right) \cdot \operatorname{det} M\left[V(H) \backslash\left\{v_{1}, v_{2}, v_{3}\right\}\right]
$$

Since the $\operatorname{wt}\left(P_{i_{s} \rightarrow j_{s}}\right)$ 's are nonzero constants and $M\left(\left\{j_{1}, j_{2}, j_{3}\right\},\left\{i_{1}, i_{2}, i_{3}\right\}\right)$ is an $(n-3)$ by $(n-3)$ submatrix of $M$, we have

$$
\Delta_{n-3}(x) \mid \operatorname{det} M\left[V(H) \backslash\left\{v_{1}, v_{2}, v_{3}\right\}\right] .
$$

By Theorem 3, if $\lambda$ is an eigenvalue of $A$ with $m_{A}(\lambda) \geq 4$, then $\lambda$ is an eigenvalue of $M\left[V(H) \backslash\left\{v_{1}, v_{2}, v_{3}\right\}\right]$ with multiplicity $m_{A}(\lambda)-3$ or more. Since the order of $M\left[V(H) \backslash\left\{v_{1}, v_{2}, v_{3}\right\}\right]$ is $m-3$, it follows that

$$
\sum_{\lambda_{j}: m_{A}\left(\lambda_{j}\right) \geq 4} 1 \leq \sum_{\lambda_{j}: m_{A}\left(\lambda_{j}\right) \geq 4}\left(m_{A}\left(\lambda_{j}\right)-3\right) \leq m-3 .
$$

Thus, by (14), 


$$
\begin{aligned}
\sum_{\lambda_{j}: m_{A}\left(\lambda_{j}\right) \geq 4}\left(m_{A}\left(\lambda_{j}\right)-2\right)= & \sum_{\lambda_{j}: m_{A}\left(\lambda_{j}\right) \geq 4}\left(m_{A}\left(\lambda_{j}\right)-3\right) \\
& +\sum_{\lambda_{j}: m_{A}\left(\lambda_{j}\right) \geq 4} 1 \leq 2 m-6 .
\end{aligned}
$$

Next, we compute the determinants of certain $(n-2)$ by $(n-2)$ submatrices of $M$ and thereby find an upper bound on $n_{3}$. Let $\left\{a_{1}, b_{1}\right\}=\left\{i_{r}, j_{r}\right\},\left\{a_{2}, b_{2}\right\}=\left\{i_{s}, j_{s}\right\}$ and $\left\{a_{3}, b_{3}\right\}=\left\{i_{t}, j_{t}\right\}$ for some $r, s$ and $t$ with $\{r, s, t\}=\{1,2,3\}$. The submatrices of concern have the form $M\left(\left\{a_{1}, a_{2}\right\},\left\{b_{1}, b_{3}\right\}\right)$.

By considering the matrix $B$ obtained from $M$ by interchanging columns $a_{2}$ and $b_{3}$, and the digraph $D\left(B\left(\left\{a_{2}\right\}\right)\right)$, a similar argument as in the proof of Corollary 7 shows that

$$
\operatorname{det}\left[M\left(\left\{a_{2}\right\},\left\{b_{3}\right\}\right)\right]\left(\left\{a_{1}\right\},\left\{b_{1}\right\}\right)= \pm \mathrm{wt}\left(P_{a_{1} \rightarrow b_{1}}\right) \operatorname{det}\left[M\left(V\left(P_{a_{1}-b_{1}}\right)\right)\right]\left(\left\{a_{2}\right\},\left\{b_{3}\right\}\right) .
$$

Let $N=M\left(V\left(P_{a_{1}-b_{1}}\right)\right)$. By Theorem 6,

$$
\operatorname{det} N\left(\left\{a_{2}\right\},\left\{b_{3}\right\}\right)= \pm \sum_{P}(-1)^{\ell(P)} \mathrm{wt}(P) \operatorname{det} N(V(P))
$$

where the sum is taken over all directed paths $P$ in $D(N)$ from $b_{3}$ to $a_{2}$. Note that $N(V(P))$ is permutationally similar to $M\left[V\left(L_{b_{2}}\right)\right] \oplus M\left[V\left(L_{a_{3}}\right)\right] \oplus M[V(H) \backslash V(P)]$ where $L_{b_{2}}$ and $L_{a_{3}}$ are the legs containing $b_{2}$ and $a_{3}$, respectively. It follows that there is a polynomial $f_{r}(x)$ such that $\operatorname{det} M\left(\left\{a_{1}, a_{2}\right\},\left\{b_{1}, b_{3}\right\}\right)$ is a constant multiple of det $M\left[V\left(L_{b_{2}}\right)\right]$ det $M\left[V\left(L_{a_{3}}\right)\right] f_{r}(x)$. Note that $f_{r}(x)$ depends only upon $s$ and $t$ and not the choice of $a_{2} \in\left\{i_{s}, j_{s}\right\}$ and $b_{3} \in\left\{i_{t}, j_{t}\right\}$. Also note that the assumption that there is a unique shortest directed path from $v_{s}$ to $v_{t}$ not through $v_{r}$ implies that $f_{r}(x)$ is a nonzero polynomial of degree at most $m-3$. Since $M\left(\left\{a_{1}, a_{2}\right\},\left\{b_{1}, b_{3}\right\}\right)$ is an $(n-2)$ by $(n-2)$ submatrix of $M$,

$$
\Delta_{n-2}(x) \mid \operatorname{det} M\left[V\left(L_{b_{2}}\right)\right] \cdot \operatorname{det} N\left[V\left(L_{a_{3}}\right)\right] \cdot f_{r}(x) .
$$

Let $\mu \in \sigma(A)$ and $m_{A}(\mu)=3$. Then, by Theorem 3, $\mu$ is a zero of $\operatorname{det} M\left[V\left(L_{b_{2}}\right)\right]$. $\operatorname{det} M\left[V\left(L_{a_{3}}\right)\right] \cdot f_{r}(x)$. If $\mu$ is an eigenvalue of neither $A\left[V\left(L_{b_{2}}\right)\right]$ nor $A\left[V\left(L_{a_{3}}\right)\right]$, then $\mu$ is a zero of $f_{r}(x)$. Note that $L_{b_{2}}$ and $L_{a_{3}}$ are arbitrary legs of $G$ incident to different $v_{i}$ 's.

If $\mu$ is not an eigenvalue of at least 4 of $A\left[L_{r}\right]$ 's, then there exist $A\left[V\left(L_{u}\right)\right]$ and $A\left[V\left(L_{v}\right)\right]$ such that $\lambda \notin \sigma\left(A\left[V\left(L_{u}\right)\right]\right)$ and $\lambda \notin \sigma\left(A\left[V\left(L_{v}\right)\right]\right)$, and $L_{u}, L_{v}$ are connected to different $v_{i}$ 's. Thus, in this case, $\mu$ is a zero of $f_{k}(x)$ for some $k \in\{1,2,3\}$. Therefore, the number, $n_{3}^{\prime}$, of such $\mu$ satisfies

$$
n_{3}^{\prime} \leq 3(m-3)
$$

Let $n_{3}^{\prime \prime}$ be the number of eigenvalues $\mu$ of $A$ with multiplicity 3 such that $\mu$ is an eigenvalue of at least 4 of $A\left[V\left(L_{r}\right)\right]$ 's. Then $n_{3}=n_{3}^{\prime}+n_{3}^{\prime \prime}$. Since $\mu$ is an eigenvalue of $A\left[V\left(L_{1}\right)\right] \oplus \cdots \oplus A\left[V\left(L_{6}\right)\right]$ with multiplicity at least 4 , and the order of $A\left[V\left(L_{1}\right)\right] \oplus$ 
$\cdots \oplus A\left[V\left(L_{6}\right)\right]$ is $6 \ell, 4 n_{3}^{\prime \prime} \leq 6 \ell$. Equivalently,

$$
n_{3}^{\prime \prime} \leq \frac{3 \ell}{2}
$$

Now, we compute a lower bound on $q(A)=q$. Note that

$$
\begin{aligned}
n=6 \ell+m=\sum_{i=1}^{q} m_{A}\left(\lambda_{i}\right) & =2 q-n_{1}+n_{3}^{\prime}+n_{3}^{\prime \prime}+\sum_{\lambda_{j}: m_{A}\left(\lambda_{j}\right) \geq 4}\left(m_{A}\left(\lambda_{j}\right)-2\right) \\
& \leq 2 q+n_{3}^{\prime}+n_{3}^{\prime \prime}+\sum_{\lambda_{j}: m_{A}\left(\lambda_{j}\right) \geq 4}\left(m_{A}\left(\lambda_{j}\right)-2\right) .
\end{aligned}
$$

By (15), (16), and (17), we have

$$
6 \ell+m \leq 2 q+3(m-3)+\frac{3 \ell}{2}+2 m-6 .
$$

Hence, by solving (18) for $q=q(A)$, we have

$$
q(A) \geq \frac{9 \ell}{4}-2 m+\frac{15}{2} .
$$

Since $A$ is an arbitrary matrix in $S(G),(19)$ implies

$$
q(G) \geq \frac{9 \ell}{4}-2 m+\frac{15}{2} .
$$

\section{Coda}

There has been some very interesting previous research on the relationship between the structure of the Jordan blocks associated with the eigenvalue 0 of a matrix and the digraph of the matrix, and that Smith Normal Form techniques were used to prove some of the results. More specifically, in the case that $A$ is a nilpotent upper triangular matrix, Gansner [6] gave necessary conditions on the sizes of the Jordan blocks of $A$ associated with 0 in terms of sizes of disjoint paths. Moreover, the necessary conditions were shown to hold for generic $A$. Hershkowitz and Schneider (see the survey [7]) extended Gansner's results to the much more difficult case of upper triangular matrices and then to the even more difficult case of arbitrary matrices. In particular, the work of Hershkowitz and Schneider gives a combinatorial formula for the maximum multiplicity of an eigenvalue among the matrices with a given zero-nonzero pattern.

There are two main differences between the previous work and this paper. First, in the present work, when studying the family of matrices with a given graph, the diagonal entries are allowed to be arbitrary. In the previous work, each diagonal entry was fixed to be zero, or nonzero. Second, and more importantly, the previous work studied the Jordan structure of single eigenvalue at a time, and the present work begins the simultaneous study of the Jordan structure of all of the eigenvalues. In this light, a general problem, that this paper only begins to address is the following: 
Given a graph $G$ (or a digraph $D$ ), determine all the possible structures of the Jordan blocks (of all multiplicities) of matrices with graph $G$ (or digraph $D$ ).

More precisely,

Given $G$ (or $D$ ) find necessary and sufficient conditions for the existence of a matrix $A$ with graph $G$ (or digraph $D$ ) such that $A$ has $q$ distinct eigenvalues, and the sizes of the Jordan blocks of $A$ corresponding to the $i$ th eigenvalue are $s_{i_{1}}, s_{i_{2}}, \ldots, s_{i_{b_{i}}}$ for $i=1,2, \ldots, q$.

A complete solution in the case that $G$ is a tree would be significant, and appears difficult.

Acknowledgements The work of In-Jae Kim was done while he was a graduate student at the University of Wyoming. The authors thank the referees for bringing the references $[6,7,15]$ to our attention and helpful comments that greatly improved the presentation.

\section{References}

1. Barioli, F., Fallat, S.M.: On two conjectures regarding an inverse eigenvalue problem for acyclic symmetric matrices. Electron. J. Linear Algebra 11, 41-50 (2004)

2. Bridges, W.G., Mena, R.: Lecture Notes. University of Wyoming, Laramie (1984)

3. Fiedler, M.: Eigenvectors of acyclic matrices. Czech. Math. J. 25, 607-618 (1975)

4. Godsil, C., Royle, G.: Algebraic Graph Theory. Springer, New York (2001)

5. Hartley, B., Hawkes, T.O.: Rings, Modules and Linear Algebra. Chapman \& Hall, London (1994)

6. Gansner, E.R.: Acyclic digraphs, Young tableaux and nilpotent matrices. SIAM J. Algebraic Discrete Methods 2, 429-440 (1981)

7. Hershkowitz, D.: Paths in directed graphs and spectral properties of matrices. Linear Algebra Appl. 212/213, 309-337 (1994)

8. Johnson, C.R., Leal Duarte, A.: The maximum multiplicity of an eigenvalue in a matrix whose graph is a tree. Linear and Multilinear Algebra 46, 139-144 (1999)

9. Johnson, C.R., Leal Duarte, A.: On the possible multiplicities of the eigenvalues of a Hermitian matrix whose graph is a tree. Linear Algebra Appl. 348, 7-21 (2002)

10. Johnson, C.R., Leal Duarte, A., Saiago, C.M.: The Parter-Wiener theorem: refinement and generalization. SIAM J. Matrix Anal. Appl. 25, 352-361 (2003)

11. Johnson, C.R., Leal Duarte, A., Saiago, C.M.: Inverse eigenvalue problems and list of eigenvalues for matrices whose graph is a tree: the case of generalized stars and double generalized stars. Linear Algebra Appl. 373, 311-330 (2003)

12. Johnson, C.R., Leal Duarte, A., Saiago, C.M., Sutton, B.D., Witt, A.J.: On the relative position of multiple eigenvalues in the spectrum of an Hermitian matrix with a given graph. Linear Algebra Appl. 363, 147-159 (2003)

13. Leal Duarte, A.: Construction of acyclic matrices from spectral data. Linear Algebra Appl. 113, 173182 (1989)

14. Leal Duarte, A., Johnson, C.R.: On the minimum number of distinct eigenvalues for a symmetric matrix whose graph is a given tree. Math. Inequal. Appl. 5, 175-180 (2002)

15. Maybee, J., Olesky, D.D., van den Driessche, P., Wiener, G.: Matrices, digraphs and determinants. SIAM J. Matrix Anal. Appl. 10, 500-519 (1989)

16. Nylen, P.M.: Minimum-rank matrices with prescribed graph. Linear Algebra Appl. 248, 303-316 (1996) 